\title{
A generalization of Gale's lemma
}

\section{Alishahi, Meysam; Hajiabolhassan, Hossein}

Published in:

Journal of Graph Theory

Link to article, DOI:

10.1002/jgt.22215

Publication date:

2017

Document Version

Peer reviewed version

Link back to DTU Orbit

Citation (APA):

Alishahi, M., \& Hajiabolhassan, H. (2017). A generalization of Gale's lemma. Journal of Graph Theory, 88(2), 337-346. https://doi.org/10.1002/jgt.22215

\section{General rights}

Copyright and moral rights for the publications made accessible in the public portal are retained by the authors and/or other copyright owners and it is a condition of accessing publications that users recognise and abide by the legal requirements associated with these rights.

- Users may download and print one copy of any publication from the public portal for the purpose of private study or research.

- You may not further distribute the material or use it for any profit-making activity or commercial gain

- You may freely distribute the URL identifying the publication in the public portal

If you believe that this document breaches copyright please contact us providing details, and we will remove access to the work immediately and investigate your claim 


\title{
A generalization of Gale's lemma
}

\author{
Meysam Alishahi $^{1}$ | Hossein Hajiabolhassan ${ }^{2,3}$ (DD
}

\author{
${ }^{1}$ School of Mathematical Sciences, Shahrood \\ University of Technology, Shahrood, Iran \\ ${ }^{2}$ Department of Applied Mathematics and \\ Computer Science, Technical University of \\ Denmark, DK-2800 Lyngby, Denmark \\ ${ }^{3}$ Department of Mathematical Sciences, \\ Shahid Beheshti University, G.C., Tehran, \\ Iran \\ Correspondence \\ Hossein Hajiabolhassan, Department of \\ Applied Mathematics and Computer Science, \\ Technical University of Denmark, DK-2800 \\ Lyngby, Denmark, and Department of Mathe- \\ matical Sciences, Shahid Beheshti University, \\ G.C., P.O. Box 19839-69411, Tehran, Iran. \\ Email:hhhaji@yahoo.com
}

\begin{abstract}
In this work, we present a generalization of Gale's lemma. Using this generalization, we introduce two sharp combinatorial lower bounds for coind $\left(\mathrm{B}_{0}(G)\right)+1$ and coind $(\mathrm{B}(G))+2$, the two classic topological lower bounds for the chromatic number of a graph $G$.
\end{abstract}

\section{K E Y W O R D S}

box complex, chromatic number of graphs, Gale's lemma

\section{1 | INTRODUCTION AND MAIN RESULTS}

\subsection{Backgrounds and motivations}

Throughout the article, for positive integers $k$ and $n$, two symbols $[n]$ and $\left(\begin{array}{c}{[n]} \\ k\end{array}\right)$ stand for the set $\{1, \ldots, n\}$ and the family of all $k$-subsets of $[n]$, respectively. For a positive integer $s$, a subset $A$ of $[n]$ is said to be an $s$-stable subset if $s \leq|i-j| \leq n-s$ for each $i \neq j \in A$. Throughout the article, the family of all $s$-stable $k$-subsets of $[n]$ is denoted by $\left(\begin{array}{c}{[n]} \\ k\end{array}\right)$. For $n \geq 2 k$, the Kneser graph $\operatorname{KG}(n, k)$ is a graph whose vertex set consists of all $k$-subsets of $[n]$ and two vertices are adjacent if their corresponding $k$ sets are disjoint. Kneser 1955 [11] proved that $\operatorname{KG}(n, k)$ can be properly colored with $n-2 k+2$ colors. He also conjectured that this is the best possible, i.e. $\chi(\operatorname{KG}(n, k)) \geq n-2 k+2$. In 1978, Lovász in a fascinating article [12], using the Borsuk-Ulam theorem, gave an affirmative answer to this conjecture.

For an integer $d \geq-1$, by the symbol $S^{d}$, we mean the $d$-dimensional sphere. For an $x \in S^{d}, H(x)$ is the open hemisphere centered at $x$, i.e. $H(x)=\left\{y \in S^{d}:\langle x, y\rangle>0\right\}$. There is a well-known lemma due to Gale [8] that asserts that for every $k \geq 1$ and every $n>2 k$, there is an $n$-set $Z \subset S^{n-2 k}$ such that for any $x \in S^{n-2 k}$, the open hemisphere $H(x)$ contains at least $k$ points of $Z$. In particular, if we identify the set $Z$ with $[n]$, then for any $x \in S^{n-2 k}$, the open hemisphere $H(x)$ contains some vertex of $\operatorname{KG}(n, k)$. Soon after the announcement of the Lovász breakthrough [12], Bárány [5] presented a short proof of the Lovász-Kneser theorem based on Gale's lemma. Next, Schrijver [16] generalized Gale's lemma by proving that there is an $n$-set $Z \subset S^{n-2 k}$ and a suitable identification of $Z$ with [ $n$ ] such that for any $x \in S^{n-2 k}$, the open hemisphere $H(x)$ contains a 2-stable subset of size at least $k$, i.e. the 
open hemisphere $H(x)$ contains some member of $\left(\begin{array}{c}{[n]} \\ k\end{array}\right)$. Using this generalization, Schrijver [16] found a specific vertex-critical subgraph of $\mathrm{KG}(n, k)$ having the same chromatic number as $\mathrm{KG}(n, k)$. This subgraph, known as the Schrijver graph $\mathrm{SG}(n, k)$, is an induced subgraph of $\mathrm{KG}(n, k)$ whose vertex set consists of all 2-stable $k$-subsets of [n]. It is worth mentioning that Greene [9] showed that Bárány's idea can be used in a more tricky way to avoid the use of Gale's lemma while Greene's trick does not seem to work for the case of Schrijver graphs, that is, Gale's lemma is still essential for a Bárány type proof of Schrijver's theorem.

\subsection{Main results}

For an $X=\left(x_{1}, \ldots, x_{n}\right) \in\{+,-, 0\}^{n}$, an alternating subsequence of $X$ is a subsequence of nonzero terms of $X$ such that each of its two consecutive members have different signs. In other words, $x_{j_{1}}, \ldots, x_{j_{m}}\left(1 \leq j_{1}<\cdots<j_{m} \leq n\right)$ is an alternating subsequence of $X$ if $x_{j_{i}} \neq 0$ for each $i \in[m]$ and $x_{j_{i}} \neq x_{j_{i+1}}$ for $i=1, \ldots, m-1$. The length of the longest alternating subsequence of $X$ is denoted by $\operatorname{alt}(X)$. We also set $\operatorname{alt}(0, \ldots, 0)=0$. Moreover, define

$$
X^{+}=\left\{j: x_{j}=+\right\} \quad \text { and } \quad X^{-}=\left\{j: x_{j}=-\right\} .
$$

Note that, by abuse of notation, we can write $X=\left(X^{+}, X^{-}\right)$.

Let $V$ be a nonempty finite set of size $n$. The signed-power set of $V$, denoted by $P_{S}(V)$, is defined as follows;

$$
P_{s}(V)=\{(A, B): A, B \subseteq V, A \cap B=\varnothing\} .
$$

For two pairs $(A, B)$ and $(C, D)$ in $P_{S}(V)$, by $(A, B) \subseteq(C, D)$, we mean $A \subseteq C$ and $B \subseteq D$. Note that $\left(P_{S}(V), \subseteq\right)$ is a partially order set (poset). A signed-increasing property $\mathcal{P}$, is a superset-closed family $\mathcal{P} \subseteq P_{s}(V)$, i.e. for any $F_{1} \in \mathcal{P}$, if $F_{1} \subseteq F_{2} \in P_{s}(V)$, then $F_{2} \in \mathcal{P}$. Clearly, for any bijection $\sigma:[n] \rightarrow V$, the map $X \mapsto X_{\sigma}=\left(\sigma\left(X^{+}\right), \sigma\left(X^{-}\right)\right.$is an identification between $\{+,-, 0\}^{n}$ and $P_{S}(V)$. Let $\sigma:[n] \rightarrow V$ be a bijection and $\mathcal{P} \subseteq P_{s}(V)$ be a signed-increasing property. Define

$$
\operatorname{alt}(\mathcal{P}, \sigma)=\max \left\{\operatorname{alt}(X): X \in\{+,-, 0\}^{n} \text { with } X_{\sigma} \notin \mathcal{P}\right\}
$$

Also, define the alternation number of $\mathcal{P}$ to be the following quantity;

$$
\operatorname{alt}(\mathcal{P})=\min \{\operatorname{alt}(\mathcal{P}, \sigma): \sigma:[n] \rightarrow V \text { is a bijection }\}
$$

Let $d \geq 0$ be an integer, $S^{d}$ be the $d$-dimensional sphere, and $Z \subset S^{d}$ be a finite set. For an $x \in$ $S^{d}$, define $Z_{x}=\left(Z_{x}^{+}, Z_{x}^{-}\right) \in P_{s}(Z)$ where $Z_{x}^{+}=H(x) \cap Z$ and $Z_{x}^{-}=H(-x) \cap Z$. Now, we are in a position to state the first main result of this article (Lemma 1), which is a generalization of Gale's lemma. The fact that the following lemma generalizes Gale's lemma will be more clarified later, see Corollary 1 and the discussion after it.

Lemma 1. Let $n$ be a positive integer, $V$ be an $n$-set, and $\sigma:[n] \rightarrow V$ be a bijection. Also, let $\mathcal{P} \subseteq P_{S}(V)$ be a signed-increasing property and set $d=n-\operatorname{alt}(\mathcal{P}, \sigma)-1$. If $d \neq-1$, then there are a multiset $Z \subset S^{d}$ of size $n$ and a suitable identification of $Z$ with $V$ such that for any $x \in S^{d}, Z_{x} \in \mathcal{P}$. In particular, for $d \geq 1, Z$ can be a set.

A hypergraph $\mathcal{H}$ is a pair $(V(\mathcal{H}), E(\mathcal{H}))$ where $V(\mathcal{H})$ is a finite nonempty set, called the vertex set of $\mathcal{H}$, and $E(\mathcal{H})$ is a family containing some nonempty distinct subsets of $V(\mathcal{H})$, called the edge set of $\mathcal{H}$. For a set $U \subseteq V(\mathcal{H})$, the induced subhypergraph $\mathcal{H}[U]$ of $\mathcal{H}$, is a hypergraph with vertex set $U$ and 
edge set $\{e \in E(\mathcal{H}): e \subseteq U\}$. A graph $G$ is a hypergraph such that each of its edges has cardinality two. A $t$-coloring of a hypergraph $\mathcal{H}$ is a map $c: V(\mathcal{H}) \rightarrow[t]$ such that for no edge $e \in E(\mathcal{H})$, we have $|c(e)|=1$. The minimum possible $t$ for which $\mathcal{H}$ admits a $t$-coloring, denoted by $\chi(\mathcal{H})$, is called the chromatic number of $\mathcal{H}$. For a hypergraph $\mathcal{H}$, the Kneser graph $\mathrm{KG}(\mathcal{H})$ is a graph whose vertex set is $E(\mathcal{H})$ and two vertices are adjacent if their corresponding edges are vertex disjoint. It is known that for any graph $G$, there are infinitely many hypergraphs $\mathcal{H}$ for which $G$ and $\operatorname{KG}(\mathcal{H})$ are isomorphic. Each of those hypergraphs $\mathcal{H}$ is called a Kneser representation of $G$. Note that if we set $K_{n}^{k}=\left([n],\left(\begin{array}{c}{[n]} \\ k\end{array}\right)\right)$ and $\widetilde{K_{n}^{k}}=\left([n],\left(\begin{array}{c}{[n]} \\ k\end{array}\right)_{2}\right)$, then $\operatorname{KG}\left(K_{n}^{k}\right)=\operatorname{KG}(n, k)$ and $\operatorname{KG}\left(\widetilde{K_{n}^{k}}\right)=\operatorname{SG}(n, k)$. The colorability defect of a hypergraph $\mathcal{H}$, denoted by $\operatorname{cd}(\mathcal{H})$, is the minimum number of vertices that should be excluded so that the induced subhypergraph on the remaining vertices is 2-colorable. Dol'nikov [7] improved Lovász's result [12] by proving that for any hypergraph $\mathcal{H}$, we have $\chi(\operatorname{KG}(\mathcal{H})) \geq \operatorname{cd}(\mathcal{H})$.

Let $\mathcal{H}=(V, E)$ be a hypergraph and $\sigma:[n] \rightarrow V(\mathcal{H})$ be a bijection. Define

$$
\operatorname{alt}(\mathcal{H}, \sigma)=\max \left\{\operatorname{alt}(X): X \in\{+,-, 0\}^{n} \text { s.t. } \max \left(\left|E\left(\mathcal{H}\left[\sigma\left(X^{+}\right)\right]\right)\right|,\left|E\left(\mathcal{H}\left[\sigma\left(X^{-}\right)\right]\right)\right|\right)=0\right\}
$$

and

$$
\operatorname{salt}(\mathcal{H}, \sigma)=\max \left\{\operatorname{alt}(X): X \in\{+,-, 0\}^{n} \text { s.t. } \min \left(\left|E\left(\mathcal{H}\left[\sigma\left(X^{+}\right)\right]\right)\right|,\left|E\left(\mathcal{H}\left[\sigma\left(X^{-}\right)\right]\right)\right|\right)=0\right\} .
$$

In other words, $\operatorname{alt}(\mathcal{H}, \sigma)($ resp. $\operatorname{salt}(\mathcal{H}, \sigma))$ is the maximum possible alt $(X)$, where $X \in\{+,-, 0\}^{n}$, such that each of (resp. at least one of) $\sigma\left(X^{+}\right)$and $\sigma\left(X^{+}\right)$contains no edge of $\mathcal{H}$. We also define

$$
\operatorname{alt}(\mathcal{H})=\min _{\sigma} \operatorname{alt}(\mathcal{H}, \sigma) \quad \text { and } \quad \operatorname{salt}(\mathcal{H})=\min _{\sigma} \operatorname{salt}(\mathcal{H}, \sigma)
$$

where the minimum is taken over all bijections $\sigma:[n] \rightarrow V(\mathcal{H})$. The present authors, using Tucker's lemma [21], introduced two tight combinatorial lower bounds for the chromatic number of $\operatorname{KG}(\mathcal{H})$ improving Dol'nikov's lower bound.

Theorem A. [1] For any hypergraph $\mathcal{H}$, we have

$$
\chi(K G(\mathcal{H})) \geq \max (|V(\mathcal{H})|-\operatorname{alt}(\mathcal{H}),|V(\mathcal{H})|-\operatorname{salt}(\mathcal{H})+1) .
$$

Let $\mathcal{H}=(V, E)$ be a hypergraph and $\sigma:[n] \rightarrow V(\mathcal{H})$ be a bijection. Note that if we set

$$
\mathcal{P}_{1}=\left\{(A, B) \in P_{S}(V): \text { at least one of } A \text { and } B \text { contains some edge of } \mathcal{H}\right\}
$$

and

$$
\mathcal{P}_{2}=\left\{(A, B) \in P_{S}(V) \text { : both of } A \text { and } B \text { contain some edges of } \mathcal{H}\right\}
$$

then $\operatorname{alt}\left(\mathcal{P}_{1}, \sigma\right)=\operatorname{alt}(\mathcal{H}, \sigma)$ and $\operatorname{alt}\left(\mathcal{P}_{2}, \sigma\right)=\operatorname{salt}(\mathcal{H}, \sigma)$. Therefore, in view of Lemma 1 , we have the next result.

Corollary 1. For a hypergraph $\mathcal{H}=(V, E)$ and a bijection $\sigma:[n] \rightarrow V(\mathcal{H})$, we have the following assertions.

a) If $d=|V|-\operatorname{alt}(\mathcal{H}, \sigma)-1$ and $\operatorname{alt}(\mathcal{H}, \sigma) \neq|V|$, then there are a multiset $Z \subset S^{d}$ of size $|V|$ and a suitable identification of $Z$ with $V$ such that for any $x \in S^{d}, H(x)$ or $H(-x)$ contains some edge of $\mathcal{H}$. In particular, for $d \geq 1, Z$ can be a set. 
b) If $d=|V|-\operatorname{salt}(\mathcal{H}, \sigma)-1$ and $\operatorname{salt}(\mathcal{H}, \sigma) \neq|V|$, then there are a multiset $Z \subset S^{d}$ of size $|V|$ and a suitable identification of $Z$ with $V$ such that for any $x \in S^{d}$, the open hemisphere $H(x)$ contains some edge of $\mathcal{H}$. In particular, for $d \geq 1, Z$ can be a set.

For two positive integers $n$ and $k$, where $n>2 k$, and for the identity bijection $I:[n] \rightarrow[n]$, one can see that $\operatorname{salt}\left(\widetilde{K_{n}^{k}}, I\right)=2 k-1$. Therefore, by the second part of Corollary 1, we have the strengthening of Gale's lemma given by Schrijver [16]: there is an $n$-subset $Z$ of $S^{n-2 k}$ and a suitable identification of $Z$ with $[n]$ such that for any $x \in S^{n-2 k}$, the open hemisphere $H(x)$ contains at least a 2-stable subset of $[n]$ with size $k$.

Note that for any graph $G$, there are several hypergraphs $\mathcal{H}$ such that $\operatorname{KG}(\mathcal{H})$ and $G$ are isomorphic. By the help of Lemma 1 and as the second main result of this article, we provide two combinatorial approximations for two important topological lower bounds for the chromatic number of a graph $G$, namely, $\operatorname{coind}\left(B_{0}(G)\right)+1$ and coind $(B(G))+2$, see [18]. The quantities coind $\left(B_{0}(G)\right)$ and coind $(B(G))$ are respectively the coindices of two box complexes $\mathrm{B}_{0}(G)$ and $\mathrm{B}(G)$ that will be defined in Section 2. It should be mentioned that the two inequalities $\chi(G) \geq \operatorname{coind}\left(B_{0}(G)\right)+1 \chi(G) \geq$ coind $(B(G))+2$ are already proved [18] and we restate them in the following theorem just to emphasize that this theorem is an improvement of Theorem A.

Theorem 1. Let $G$ be a graph and $\mathcal{H}=(V, E)$ be a hypergraph such that $K G(\mathcal{H})$ and $G$ are isomorphic. Then the following inequalities hold;

a) $\chi(G) \geq \operatorname{coind}\left(B_{0}(G)\right)+1 \geq|V(\mathcal{H})|-\operatorname{alt}(\mathcal{H})$,

b) $\chi(G) \geq \operatorname{coind}(B(G))+2 \geq|V(\mathcal{H})|-\operatorname{salt}(\mathcal{H})+1$.

Note that, in addition to presenting another proof for Theorem A, Theorem 1 also reveals a new way to estimate coind $\left(B_{0}(G)\right)$ and coind $(B(G))$ for a graph $G$ as well. Also, it is worth noting that the results in [1-3] confirm that we can evaluate the chromatic number of some family of graphs by computing alt(-) or salt(-) for an appropriate choice of a hypergraph, while it seems that it is not easy to directly determine the values of coind $\left(B_{0}(-)\right)+1$ and coind $(B(-))+2$ for these graphs by topological methods.

Remark. Motivated by Corollary 1, we can assign to any hypergraph $\mathcal{H}$ two topological parameters; the dimension of $\mathcal{H}$ and the strong dimension of $\mathcal{H}$, denoted respectively by $\operatorname{dim}(\mathcal{H})$ and $\operatorname{sdim}(\mathcal{H})$. The dimension of $\mathcal{H}$ (resp. strong dimension of $\mathcal{H}$ ) is the maximum integer $d \geq-1$ such that the vertices of $\mathcal{H}$ can be identified with a multiset $Z \subset S^{d}$ so that for any $x \in S^{d}$, at least one of (resp. both of ) open hemispheres $H(x)$ and $H(-x)$ contains some edge of $\mathcal{H}$. In view of Corollary 1 and with a proof similar to the one of Theorem 1, one can prove the following corollary.

Corollary 2. For a hypergraph $\mathcal{H}$, we have the following inequalities;

a) $\operatorname{coind}\left(\boldsymbol{B}_{0}(K G(\mathcal{H}))\right) \geq \operatorname{dim}(\mathcal{H}) \geq|V(\mathcal{H})|-\operatorname{alt}(\mathcal{H})-1$,

b) $\operatorname{coind}(B(K G(\mathcal{H}))) \geq \operatorname{sdim}(\mathcal{H}) \geq|V(\mathcal{H})|-\operatorname{salt}(\mathcal{H})-1$.

This article is organized as follows. Section 2 contains a brief review of elementary but essential preliminaries and definitions that will be needed throughout the article. Section 3 is devoted to the proof of Lemma 1 and Theorem 1. Also, in this section, as an application of the generalization of Gale's lemma (Lemma 1), we reprove a result by Chen [6] concerning the multichromatic number of stable Kneser graphs. 


\section{2 | TOPOLOGICAL PRELIMINARIES}

This section is devoted to a brief review of some basic tools that we will need elsewhere in the article. However, we assume basic knowledge in topological combinatorics. More details on that topic can be found for instance in the book by Matoušek [13] or in the article by Simonyi and Tardos [17]. A $\mathbb{Z}_{2}$-space is a pair $(T, v)$, where $T$ is a topological space and $v$ is an involution, i.e. $v: T \rightarrow T$ is a continuous map such that $v^{2}$ is the identity map. For an $x \in T$, two points $x$ and $v(x)$ are called antipodal. The $\mathbb{Z}_{2}$-space $(T, v)$ is called free if there is no $x \in T$ such that $v(x)=x$. For instance, one can see that the unit sphere $S^{d} \subset \mathbb{R}^{d+1}$ with the involution given by the antipodal map $-: x \mapsto-x$ is free. For two $\mathbb{Z}_{2}$-spaces $\left(T_{1}, v_{1}\right)$ and $\left(T_{2}, v_{2}\right)$, a continuous map $f: T_{1} \rightarrow T_{2}$ is called a $\mathbb{Z}_{2}$-map if $f \circ v_{1}=v_{2} \circ f$. The existence of such a map is denoted by $\left(T_{1}, v_{1}\right) \stackrel{\mathbb{Z}_{2}}{\rightarrow}\left(T_{2}, v_{2}\right)$. For a $\mathbb{Z}_{2}$-space $(T, v)$, we define the $\mathbb{Z}_{2}$-index and $\mathbb{Z}_{2}$-coindex of $(T, v)$, respectively, as

$$
\operatorname{ind}(T, v)=\min \left\{d \geq 0(T, v) \stackrel{\mathbb{Z}_{2}}{\rightarrow}\left(S^{d},-\right)\right\}
$$

and

$$
\operatorname{coind}(T, v)=\max \left\{d \geq 0\left(S^{d},-\right) \stackrel{\mathbb{Z}_{2}}{\rightarrow}(T, v)\right\}
$$

If for any $d \geq 0$, there is no $(T, v) \stackrel{\mathbb{Z}_{2}}{\rightarrow}\left(S^{d},-\right)$, then we set ind $(T, v)=\infty$. Also, if $(T, v)$ is not free, then $\operatorname{ind}(T, v)=\operatorname{coind}(T, v)=\infty$.

For simplicity of notation, when the involution is understood from the context, we speak about $T$ rather than the pair $(T, v)$; also, we set $\operatorname{ind}(T, v)=\operatorname{ind}(T)$ and $\operatorname{coind}(T, v)=\operatorname{coind}(T)$. Throughout the article, we endow the unit sphere $S^{d} \subset \mathbb{R}^{d+1}$ with the involution given by the antipodal map. Note that if $T_{1} \stackrel{\mathbb{Z}_{2}}{\rightarrow} T_{2}$, then ind $\left(T_{1}\right) \leq \operatorname{ind}\left(T_{2}\right)$ and coind $\left(T_{1}\right) \leq \operatorname{coind}\left(T_{2}\right)$. Two $\mathbb{Z}_{2}$-spaces $T_{1}$ and $T_{2}$ are $\mathbb{Z}_{2^{-}}$ equivalent, denoted by $T_{1} \stackrel{\mathbb{Z}_{2}}{\longrightarrow} T_{2}$, if $T_{1} \stackrel{\mathbb{Z}_{2}}{\rightarrow} T_{2}$ and $T_{2} \stackrel{\mathbb{Z}_{2}}{\rightarrow} T_{1}$. In particular, $\mathbb{Z}_{2}$-equivalent spaces have the same index and also same coindex.

In the following, we introduce the concept of simplicial complex that provides a bridge between combinatorics and topology. A simplicial complex can be viewed as a combinatorial object, called abstract simplicial complex, or as a topological space, called geometric simplicial complex. Here, we just review the definition of an abstract simplicial complex. However, it should be mentioned that we can assign a geometric simplicial complex to an abstract simplicial complex, called its geometric realization, and vice versa. An abstract simplicial complex is a pair $L=(V, K)$, where $V$ (the vertex set of $L$ ) is a set and $K \subseteq 2^{V}$ (the set of simplices of $L$ ) is a hereditary collection of subsets of $V$, i.e. if $A \in K$ and $B \subseteq A$, then $B \in K$. Any set $A \in K$ is called a simplex of $L$. The geometric realization of an abstract simplicial complex $L$ is denoted by $\|L\|$. For two abstract simplicial complexes $L_{1}=\left(V_{1}, K_{1}\right)$ and $L_{2}=\left(V_{2}, K_{2}\right)$, a simplicial map $f: L_{1} \rightarrow L_{2}$ is a map from $V_{1}$ to $V_{2}$ preserving the simplices, i.e. if $A \in K_{1}$, then $f(A) \in K_{2}$. A simplicial involution is a simplicial map $v: L \rightarrow L$ such that $v^{2}$ is the identity map. A simplicial $\mathbb{Z}_{2}$-complex is a pair $(L, v)$ where $L$ is a simplicial complex and $v: L \rightarrow L$ is a simplicial involution. A simplicial complex $(L, v)$ is called free if there is no simplex $A$ of $L$ such that $v(A)=A$. For two simplicial $\mathbb{Z}_{2}$-complexes $\left(L_{1}, v_{1}\right)$ and $\left(L_{2}, v_{2}\right)$, the map $f: L_{1} \rightarrow L_{2}$ is called a simplicial $\mathbb{Z}_{2}$-map if $f$ is a simplicial map and $f \circ v_{1}=v_{2} \circ f$. The existence of a simplicial $\mathbb{Z}_{2}$-map $f: L_{1} \rightarrow L_{2}$ implies the existence of a continuous $\mathbb{Z}_{2}$-map $\|f\|:\left\|L_{1}\right\| \stackrel{\mathbb{Z}_{2}}{\rightarrow}\left\|L_{2}\right\|$ that is called the geometric realization of $f$. We respectively use ind $(L)$ and $\operatorname{coind}(L)$ to refer to ind $(\|L\|)$ and coind $(\|L\|)$. 
The existence of a homomorphism between two graphs is an important and generally challenging problem in graph theory. In particular, in general, it is a hard task to determine the chromatic number of a graph $G$. In the following, we assign some free simplicial $\mathbb{Z}_{2}$-complexes to graphs in such a way that graph homomorphisms give rise to $\mathbb{Z}_{2}$-maps of the corresponding complexes. For a graph $G=(V(G), E(G))$ and two disjoint subsets $A, B \subseteq V(G)$, define $G[A, B]$ to be the induced bipartite subgraph of $G$ whose parts are $A$ and $B$, that is, a subgraph of $G$ whose vertex set is $A \cup B$ and edge set is $\{u v \in E(G): u \in A$ and $v \in B\}$.

Box Complex. For a graph $G=(V(G), E(G))$ and a subset $A \subseteq V(G)$, set

$$
\mathrm{CN}(A)=\{v \in V(G): a v \in E(G) \text { for all } a \in A\} \subseteq V(G) \backslash A \text {. }
$$

The box complex of a graph $G$, denoted by $B(G)$, is a free simplicial $\mathbb{Z}_{2}$-complex with vertex set $V(G) \uplus V(G)=V(G) \times[2]$ and the following set of simplices

$\{A \uplus B: A, B \subseteq V(G), A \cap B=\varnothing, G[A, B]$ is complete bipartite and $\mathrm{CN}(A) \neq \varnothing \neq \mathrm{CN}(B)\}$.

Also, one can consider another box complex $B_{0}(G)$ with vertex set $V(G) \uplus V(G)=V(G) \times[2]$ and the following set of simplices

$\{A \uplus B: A, B \subseteq V(G), A \cap B=\varnothing, G[A, B]$ is complete bipartite $\}$.

The involution on $B(G)$ (resp. $B_{0}(G)$ ) is given by interchanging the two copies of $V(G)$; that is, $(v, 1) \mapsto(v, 2)$ and $(v, 2) \mapsto(v, 1)$ for any $v \in V(G)$. In view of these involutions, one can consider $\|B(G)\|$ and $\left\|B_{0}(G)\right\|$ as free $\mathbb{Z}_{2}$-spaces. One can check that any graph homomorphism $G \rightarrow H$ implies that there are two simplicial $\mathbb{Z}_{2}$-maps $B(G) \stackrel{\mathbb{Z}_{2}}{\rightarrow} B(H)$ and $B_{0}(G) \stackrel{\mathbb{Z}_{2}}{\rightarrow} B_{0}(H)$; and consequently, ind $(B(G)) \leq \operatorname{ind}(B(H))$, coind $(B(G)) \leq \operatorname{coind}(B(H))$, ind $\left(B_{0}(G)\right) \leq \operatorname{ind}\left(B_{0}(H)\right)$, and coind $\left(B_{0}(G)\right) \leq \operatorname{coind}\left(B_{0}(H)\right)$. It is known that $B\left(K_{n}\right)$ and $B_{0}\left(K_{n}\right)$ are $\mathbb{Z}_{2}$-equivalent to $S^{n-2}$ and $S^{n-1}$, respectively. Hence, $\chi(G) \geq \operatorname{ind}(B(G))+2 \geq \operatorname{coind}(B(G))+2$ and $\chi(G) \geq \operatorname{ind}\left(B_{0}(G)\right)+1 \geq$ coind $\left(B_{0}(G)\right)+1$. Indeed, it is known that (see $\left.[13,17,18]\right)$

$$
\chi(G) \geq \operatorname{ind}(B(G))+2 \geq \operatorname{ind}\left(B_{0}(G)\right)+1 \geq \operatorname{coind}\left(B_{0}(G)\right)+1 \geq \operatorname{coind}(B(G))+2 .
$$

For more about different simplicial complexes assigned to graphs and their relationships, one can see [14].

\section{3 | PROOF OF MAIN RESULTS}

This section is devoted to the proofs of main results. It should be mentioned that the following proof is based on an idea similar to that used in an interesting proof of Ziegler for Gale's lemma (see page 67 in [13]).

Proof of Lemma 1. For simplicity of notation, assume that $V=\left\{v_{1}, \ldots, v_{n}\right\}$ where $\sigma(i)=v_{i}$. Consider the following curve

$$
\gamma=\left\{\left(1, t, t^{2}, \ldots, t^{d}\right) \in \mathbb{R}^{d+1}: t \in \mathbb{R}\right\}
$$

and set $W=\left\{w_{1}, w_{2}, \ldots, w_{n}\right\}$, where $w_{i}=\gamma(i)$ for $i=1,2, \ldots, n$. Now, let $Z=\left\{z_{1}, z_{2}, \ldots, z_{n}\right\} \subseteq S^{d}$ be a set such that $z_{i}=(-1)^{i} \frac{w_{i}}{\left\|w_{i}\right\|}$ for any $1 \leq i \leq n$. Note that if $d \geq 1$, then $Z$ is a set. Consider 
the identification between $V$ and $Z$ such that $v_{i} \in V$ is identified with $z_{i}$ for any $1 \leq i \leq n$. It can be checked that every hyperplane of $\mathbb{R}^{d+1}$ passing trough the origin intersects $\gamma$ in no more than $d$ points. Moreover, if a hyperplane intersects the curve in exactly $d$ points, then the hyperplane cannot be tangent to the curve; and consequently, at each intersection point, the curve passes from one side of the hyperplane to the other side.

In what follows, for any $y \in S^{d}$, we will show that $Z_{y} \in \mathcal{P}$ completing the proof. On the contrary, suppose that there is a $y \in S^{d}$ such that $Z_{y} \notin \mathcal{P}$. Let $h$ be the hyperplane passing trough the origin that contains the boundary of $H(y)$. We can move this hyperplane continuously to a position such that it still contains the origin and has exactly $d$ points of $W=\left\{w_{1}, w_{2}, \ldots, w_{n}\right\}$ while during this movement no points of $W$ crosses from one side of $h$ to the other side. Consequently, during the aforementioned movement, no points of $Z=\left\{z_{1}, z_{2}, \ldots, z_{n}\right\}$ crosses from one side of $h$ to the other side. Hence, at each of these intersections, $\gamma$ passes from one side of $h$ to the other side. Let $h^{+}$and $h^{-}$be two open half-spaces determined by the hyperplane $h$. Now consider $X=\left(x_{1}, x_{2}, \ldots, x_{n}\right) \in\{+,-, 0\}^{n} \backslash\{\mathbf{0}\}$ such that

$$
x_{i}=\left\{\begin{array}{ll}
0 & \text { if } w_{i} \text { is on } h \\
+ & \text { if } w_{i} \text { is in } h^{+} \text {and } i \text { is even } \\
+ & \text { if } w_{i} \text { is in } h^{-} \text {and } i \text { is odd } \\
- & \text { otherwise. }
\end{array} .\right.
$$

Assume that $x_{i_{1}}, x_{i_{2}}, \ldots, x_{i_{n-d}}$ are nonzero entries of $X$, where $i_{1}<i_{2}<\cdots<i_{n-d}$. It is easy to check that any two consecutive terms of $x_{i_{j}}$ 's have different signs. Since $X$ has $n-d=\operatorname{alt}(\mathcal{P}, \sigma)+1$ nonzero entries, we have $\operatorname{alt}(X)=\operatorname{alt}(-X)=\operatorname{alt}(\mathcal{P}, \sigma)+1$; and therefore, both $X_{\sigma}$ and $(-X)_{\sigma}$ are in $\mathcal{P}$. Also, one can see that either $X_{\sigma} \subseteq Z_{y}$ or $(-X)_{\sigma} \subseteq Z_{y}$. Therefore, since $\mathcal{P}$ is a signed-increasing property, we have $Z_{y} \in \mathcal{P}$ that is a contradiction.

Multichromatic Number of Stable Kneser Graphs. For positive integers $n, k$, and $s$, the $s$-stable Kneser graph $\operatorname{KG}(n, k)_{s}$ is an induced subgraph of $\operatorname{KG}(n, k)$ whose vertex set is $\left(\begin{array}{c}{[n]} \\ k\end{array}\right)_{s}$. In other words, $\operatorname{KG}(n, k)_{s}=\operatorname{KG}\left([n],\left(\begin{array}{c}{[n]} \\ k\end{array}\right)_{s}\right)$. The chromatic number of stable Kneser graphs has been studied in several articles, for instance see [4,10,15]. Meunier [15] posed a conjecture about the chromatic number of stable Kneser hypergraphs that is a generalization of a conjecture of Alon et al. [4]. Restricting to Kneser graphs rather than Kneser hypergraphs, Meunier's conjecture asserts that the chromatic number of $\mathrm{KG}(n, k)_{s}$ is $n-s(k-1)$ for $n \geq s k$ and $s \geq 2$. Clearly, in view of Schrijver's result [16], this conjecture is true for $s=2$. Moreover, for $s \geq 4$ and $n$ sufficiently large, Jonsson [10] gave an affirmative answer to the graph case of Meunier's conjecture.

For two positive integers $m$ and $n$ with $n \geq m$, an $m$-fold $n$-coloring of a graph $G$ is a homomorphism from $G$ to $\operatorname{KG}(n, m)$. The $m$-th multichromatic number of a graph $G$, denoted by $\chi_{m}(G)$, is defined as follows

$$
\chi_{m}(G)=\min \{n: G \rightarrow \operatorname{KG}(n, m)\}
$$

Note that $\chi_{1}(G)=\chi(G)$. An $m$-fold $n$-coloring of a graph $G$ is called a multicoloring of $G$ with color set $[n]$. The following conjecture of Stahl [19] has received a considerable attention in the literature.

Conjecture A. ([19]) For positive integers $m, n$, and $k$ with $n \geq 2 k$, we have

$$
\chi_{m}(\mathrm{KG}(n, k))=\left\lceil\frac{m}{k}\right\rceil(n-2 k)+2 m .
$$


Stahl [20] proved the accuracy of this conjecture for $k=2,3$ and arbitrary values of $m$. Chen [6] studied the multichromatic number of $s$-stable Kneser graphs and generalized Schrijver's result. In what follows, as an application of Lemma 1, we present another proof of Chen's result. Note that, as a special case of Chen's result, we have the chromatic number of $s$-stable Kneser graphs provided that $s$ is even.

Theorem B. [6] For positive integers $n, k$, and $s$ with $n \geq s k$, if $s$ is an even integer and $k \geq m$, then $\chi_{m}\left(\mathrm{KG}(n, k)_{s}\right)=n-s k+s m$.

Proof. It is straightforward to check that $\chi_{m}\left(\mathrm{KG}(n, k)_{s}\right) \leq n-s k+s m$. For a proof of this observation, we refer the reader to [6]. Therefore, it is enough to show $\chi_{m}\left(\operatorname{KG}(n, k)_{s}\right) \geq n-s k+s m$. For the set $[n]$, let $\mathcal{P}=\mathcal{P}(n, k, s) \subseteq P_{s}([n])$ be a signed-increasing property such that $(A, B) \in \mathcal{P}$ if both $A$ and $B$ contain at least $\frac{s}{2}$ pairwise disjoint $s$-stable $k$-subsets of $[n]$. One can see that $\operatorname{alt}(\mathcal{P}, I)=s k-1$ where $I:[n] \rightarrow[n]$ is the identity bijection. Thus, by Lemma 1 , for $d=n-s k$, there exists a multiset $Z \subset S^{d}$ of size $n$ such that under a suitable identification of $Z$ with $V$, for any $x \in S^{d}$, we have $Z_{x} \in \mathcal{P}$. In other words, for any $x \in S^{d}$, the open hemisphere $H(x)$ contains at least $\frac{S}{2}$ pairwise disjoint vertices of $\mathrm{KG}(n, k)_{s}$. Now let $c: V\left(\mathrm{KG}(n, k)_{s}\right) \rightarrow\left(\begin{array}{l}C \\ m\end{array}\right)$ be an $m$-fold $C$-coloring of $\mathrm{KG}(n, k)_{s}$. For each $i \in\{1,2, \ldots, C-s m+1\}$, define $A_{i}$ to be a set consisting of all $x \in S^{d}$ such that $H(x)$ contains some vertex $v$ of $\operatorname{KG}(n, k)_{S}$ having the color $i$ in its assigned color set, i.e. $i \in c(v)$. Furthermore, define $A_{C-s m+2}=S^{d} \backslash \bigcup_{i=1}^{C-s m+1} A_{i}$. One can check that each $A_{i}$ contains no pair of antipodal points, i.e. $A_{i} \cap\left(-A_{i}\right)=\varnothing$; and also, for each $i \in\{1,2, \ldots, C-s m+1\}, A_{i}$ is an open subset of $S^{d}$ and $A_{C-s m+2}$ is thus closed. Note that the Borsuk-Ulam theorem asserts that for any covering of $S^{d}$ by $d+1$ sets $B_{1}, \ldots, B_{d+1}$, each $B_{i}$ open or closed, there exists an $i$ such that $B_{i}$ contains a pair of antipodal points. Accordingly, we must have $C-s m+2 \geq d+2=n-s k+2$ that completes the proof.

In what follows, in view of Lemma 1 and with a similar approach as in proof of Proposition 8 of [17], we prove Theorem 1 .

Proof of Theorem 1. Let $\sigma:[n] \rightarrow V(\mathcal{H})$ be an arbitrary bijection. To prove the first part, set $d=$ $|V|-\operatorname{alt}(\mathcal{H}, \sigma)-1$. In view of inequalities of (1) and the definition of alt $(\mathcal{H})$, it is sufficient to prove that coind $\left(B_{0}(G)\right)+1 \geq|V|-\operatorname{alt}(\mathcal{H}, \sigma)$. If $d \leq 0$, then the assertion follows from the definition of coindex. Hence, suppose $d \geq 1$. Now in view of Corollary 1, there exists an $n$-set $Z \subset S^{d}$ and an identification of $Z$ with $V$ such that for any $x \in S^{d}$, at least one of open hemispheres $H(x)$ and $H(-x)$ contains some edge of $\mathcal{H}$.

For any vertex $A$ of $\operatorname{KG}(\mathcal{H})$ and any $x \in S^{d}$, define $D_{A}(x)$ to be the smallest distance of a point in $A \subset S^{d}$ from the set $S^{d} \backslash H(x)$. Note that $D_{A}(x)>0$ if and only if $H(x)$ contains $A$. Define

$$
D(x)=\sum_{A \in E}\left(D_{A}(x)+D_{A}(-x)\right)
$$

Since, for any $x \in S^{d}$, at least one of $H(x)$ and $H(-x)$ contains some edge of $\mathcal{H}$, we have $D(x)>0$. Thus, the map

$$
f(x)=\frac{1}{D(x)}\left(\sum_{A \in E} D_{A}(x)\|(A, 1)\|+\sum_{A \in E} D_{A}(-x)\|(A, 2)\|\right)
$$

is a $\mathbb{Z}_{2}$-map from $S^{d}$ to $\left\|B_{0}(\operatorname{KG}(\mathcal{H}))\right\|$. It implies coind $\left(B_{0}(G)\right) \geq d$.

b) To prove the second part, set $d=n-\operatorname{salt}(\mathcal{H}, \sigma)-1$. In view of the inequalities of (1) and the definition of $\operatorname{salt}(\mathcal{H})$, it is sufficient to prove that coind $(B(G))+2 \geq|V|-\operatorname{salt}(\mathcal{H}, \sigma)+1$. If $d \leq 0$, then 
the assertion follows from the definition of coindex. Hence, suppose $d \geq 1$. Now in view of Corollary 1, there is an $n$-set $Z \subset S^{d}$ and an identification of $Z$ with $V$ such that for any $x \in S^{d}$, the open hemisphere $H(x)$ contains some edge of $\mathcal{H}$. Define $D(x)=\sum_{A \in E} D_{A}(x)$. Since the open hemisphere $H(x)$ contains some edge of $\mathcal{H}$, we have $D(x)>0$ for each $x \in S^{d}$. Thus, the map

$$
f(x)=\frac{1}{2 D(x)} \sum_{A \in E} D_{A}(x)\|(A, 1)\|+\frac{1}{2 D(-x)} \sum_{A \in E} D_{A}(-x)\|(A, 2)\|
$$

is a $\mathbb{Z}_{2}$-map from $S^{d}$ to $\|B(\operatorname{KG}(\mathcal{H}))\|$. This implies coind $(B(G)) \geq d$.

\section{ACKNOWLEDGMENT}

The authors would like to express their deepest gratitude to Professor Carsten Thomassen for his insightful comments. They also thank the reviewers for all their remarks that helped in improving the presentation of the article. Moreover, they appreciate the detailed valuable comments of Dr. Saeed Shaebani. The research of Meysam Alishahi was in part supported by a grant from IPM (No. 96050013). The research of Hossein Hajiabolhassan was partially supported by ERC advanced grant GRACOL. Moreover, they would like to thank Skype for sponsoring their endless conversations in two countries.

\section{ORCID}

Hossein Hajiabolhassan (iD http://orcid.org/0000-0001-8146-9764

\section{REFERENCES}

[1] M. Alishahi and H. Hajiabolhassan, On the chromatic number of general Kneser hypergraphs, J. Combin. Theory, Ser. B 115 (2015), 186-209.

[2] M. Alishahi and H. Hajiabolhassan, On the chromatic number of matching graphs, ArXiv e-prints, 2015.

[3] M. Alishahi and H. Hajiabolhassan, Chromatic number via Turán number, Discrete Math. 340(10) (2017), 23662377.

[4] N. Alon, L. Drewnowski, and T. Łuczak, Stable Kneser hypergraphs and ideals in $\mathbb{N}$ with the Nikodým property, Proc. Amer. Math. Soc. 137(2) (2009), 467-471.

[5] I. Bárány, A short proof of Kneser's conjecture, J. Combin. Theory Ser. A 25(3) (1978), 325-326.

[6] P.-A. Chen, On the multichromatic number of s-stable Kneser graphs, J. Graph Theory 79(3) (2015), $233-248$.

[7] V. L. Dol'nikov, A combinatorial inequality, Sibirsk. Mat. Zh. 29(3) (1988), 53-58, 219.

[8] D. Gale, Neighboring vertices on a convex polyhedron. In Linear inequalities and related system, Annals of Mathematics Studies, no. 38, . Princeton University Press, Princeton, NJ, 1956, pp. 255-263.

[9] J. E. Greene, A new short proof of Kneser's conjecture, Am. Math. Monthly 109(10) (2002), 918-920.

[10] J. Jonsson, On the chromatic number of generalized stable Kneser graphs. manuscript, 2012. https://people.kth.se/ jakobj/doc/submitted/stablekneser.pdf

[11] M. Kneser, Ein Satz über abelsche Gruppen mit Anwendungen auf die Geometrie der Zahlen, Math. Z. 61 (1955), 429-434.

[12] L. Lovász, Kneser's conjecture, chromatic number, and homotopy, J. Combin. Theory Ser. A 25(3) (1978), 319-324.

[13] J. Matoušek, Using the Borsuk-Ulam theorem. Universitext. Springer-Verlag, Berlin, 2003. Lectures on topological methods in combinatorics and geometry, Written in cooperation with Anders Björner and Günter M. Ziegler. 
[14] J. Matoušek and G. M. Ziegler, Topological lower bounds for the chromatic number: A hierarchy, Jahresber. Deutsch. Math.-Verein. 106(2) (2004), 71-90.

[15] F. Meunier, The chromatic number of almost stable Kneser hypergraphs, J. Combin. Theory Ser. A 118(6) (2011), 1820-1828.

[16] A. Schrijver, Vertex-critical subgraphs of Kneser graphs, Nieuw Arch. Wisk. (3) 26(3) (1978), 454-461.

[17] G. Simonyi and G. Tardos, Local chromatic number, Ky Fan's theorem and circular colorings, Combinatorica 26(5) (2006), 587-626.

[18] G. Simonyi, G. Tardos, and S. T. Vrećica, Local chromatic number and distinguishing the strength of topological obstructions, Trans. Amer. Math. Soc. 361(2) (2009), 889-908.

[19] S. Stahl, n-tuple colorings and associated graphs, J. Combin. Theory Ser. B 20(2) (1976), 185-203.

[20] S. Stahl, The multichromatic numbers of some Kneser graphs, Discrete Mathematics 185 (1998), $287-291$.

[21] A. W. Tucker, Some topological properties of disk and sphere. In Proc. First Canadian Math. Congress, Montreal, 1945, pp. 285-309. University of Toronto Press, Toronto, 1946.

How to cite this article: Alishahi M, Hajiabolhassan, H. A generalization of Gale's lemma. $J$ Graph Theory. 2017; 00:1-10. https://doi.org/10.1002/jgt.22215 